\title{
Consensus Problems on Small-world Networks with time delay
}

\author{
Jinjin $\mathrm{SHI}^{1, \text { a }}$, Lei $\mathrm{GU}^{2, \mathrm{~b}}$ \\ ${ }^{1}$ Shanghai Hyron Software, Shanghai, 200231,China \\ ${ }^{2}$ Shanghai Research Institute of China Telecom Corporation Limited, Shanghai, 200122,China \\ aemail: shijinjincg@163.com, bemail:gehirn@sina.com
}

Keywords: Consensus Problem; Small-world Network; Time Delay; Spectrum Radius; Laplacian matrix

\begin{abstract}
In this paper, we study the consensus problem on small-world network with time delay. In particular, we give the sufficient condition of the consensus problem reaching consensus base on a rigorous spectral analysis for the Laplacian matrix of small-world networks. Closed-form upper bound for the spectral radius for Watts-Strogatz type small-world network is presented. These general closed-form analytical results build the relationship between network topological characteristic and the network dynamical characteristic of time delay. Applying these results we can control the time delay given the network while we can also design the required small-world topology given the target time delay. Simulation results verify our analysis and close-form formula behave effectively in the design of the efficient small-world network with time delay robustness.
\end{abstract}

\section{Introduction}

Recently much attention has been focused on the on social networks theory and related dynamical behaviors. Social networks are well known to exhibit the small-world phenomenon, which comes from the observation that individuals are often linked by a short chain of acquaintances. Specifically, from Milgram's 1967 experiment [1], the average message delivery steps of United States is discover to be six, known as six degree of separation. This phenomenon in social networks has a wide application to shorten delay, improving spectral efficiency and energy efficiency. Watts and Strogatz [2] further certify that the average distance can become dramatically shorten with just few random linkages and establish the small-world models. Moveover, Newman [3] provides a comprehensive study in network structure and functions from complex networks perspective. Gu, Huang and Zhang [4] proposed a generalized small-world network model and proved the model possesses large clustering coefficient and small diameter.

The consensus problem [5] [6] for a network dynamics means to reach a common aggreement of all agents in the network, where the agents act in coordination with the others through an information network. The underlying topology of network plays an important role in the analysis of convergence speed for the consensus problem [7] [8] [9]. Gu, Zhang and Zhou [10] showed that in small-world network, the consensus speed is accelerated and become ultrafast which is first discovered by Olfati-Saber [11]. However the consensus problem with delay is much more complicated. In [8], Olfati-Saber shows that the consensus condition is much more strict for the one with delay comparing with the one without delay. Moreover the consensus problem with delay on the small-world topology still remains open.

In this paper, we study the consensus problem on Watts-Strogatz type small-world network with time delay. In particular, we give the sufficient condition of the consensus problem reaching consensus base on a rigorous spectral analysis for the Laplacian matrix of small-world networks.

The rest of the paper is organized as follows: In next section, we review some preliminary results on the small-world networks and give topological analysis on Watts-Strogatz model. The third section illustrates the dynamical behavior of consensus problem with time delay on Watts-Strogatz network and provides with our main analytical results. In the last section, we include some numerical results and our conclusion. 


\section{Small-world network and The Wattz-Strogatz model}

It was discovered that there are two important characterizations: i.e., large clustering coefficient, and the small world phenomenon (small diameter, or short average distance) in the real complex networks. The small world effect may be dated back the famous experiments carried out by Stanley Milgram [1] in the 1960s, in which letters passed from person to person were able to reach a designated target individual in only a small number of steps. In order to describe complex networks with the two properties, Watts and Strogatz [2] [12]in 1998 proposed a famous small-world network model which possesses both small average distance and large clustering coefficient by simulation if the wired probability $p \in\left[p_{1}, p_{2}\right]$.

[a]

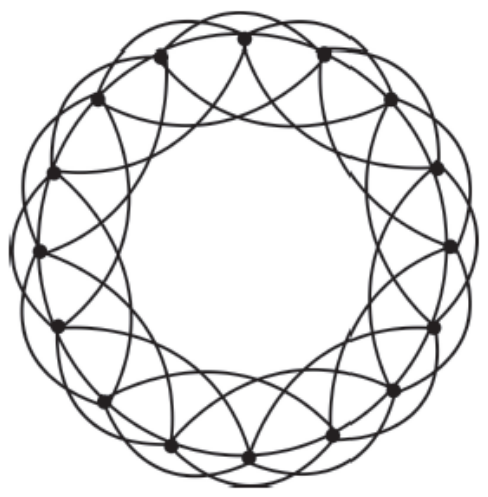

[b]

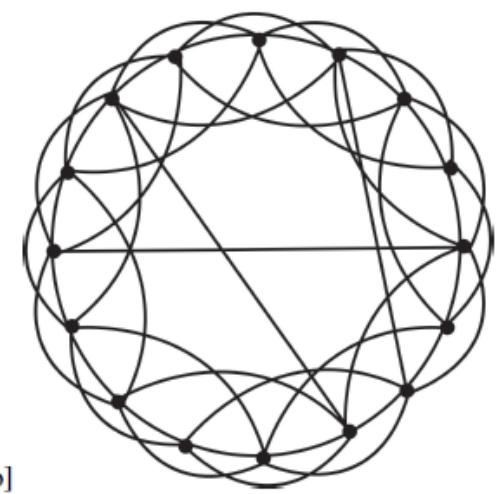

Fig. 1 Watts-Strogatz network model, [a]: the regular circulant graph; [b]: WS model with p=0.1 rewiring probability

The small-world network proposed by Watts and Strogatz as shown in Figure 1 can be described as follows:

Let $C_{n, k}$ be a $2 k$-regular circulant graph (or network) with vertex set $V\left(C_{n, k}\right)=\left\{v_{1}, v_{2}, \ldots, v_{n}\right)$ and edge set $e\left(C_{n, k}\right)=\left\{\left(v_{i}, v_{i+t}\right), 1 \leq i \leq n, 1 \leq t \leq k\right\}(i+t \equiv j \bmod n$, if $i+t>n)$. Then one rewires every edge of $C_{n, k}$ with probability $p$ by changing one end of an edge uniformly at random. No loops and multi-edges are allowed. The importance of the Watts and Strogatz' model is due to the fact that this model, roughly speaking, possesses both large clustering coefficient and small Diameter [2] [12] when $k=\Theta(\log n)$. In the following, we simply consider the Watts-Strogatz small-world model with $k=c \log n$ where $c>0$. In order to examine the graph topological property, we need the following Chernoff inequality for binomial random variables.

Lemma 1: (Chernoff inequality)[4] Let $X=\operatorname{Bin}(n, p)$ and set $\mu=n p$ then

$$
\operatorname{Pr}(X>\mu+a)<\exp \left(-\frac{a^{2}}{2(\mu+a / 3)}\right) \text {. }
$$

Now we are ready to provide the result of maximum degree of Watts-Strogatz network which will be further applied in the network dynamic system.

Lemma 2: Let $k=c \log n$. Then the maximum degree $\Delta$ of the Watts-Strogatz network $W S(n, k, p) \quad$ has an upper bound

$$
(2+p) c \log n+\left(\frac{1}{3}+\sqrt{\frac{1}{9}+2 c}\right) p \log n .
$$

\section{Proof:}

We consider to separate the inner degree and outer degree, as following the construction of the 
model, the outer degree is keeping fixed to be $k$ while the inner degree is the sum of two parts. One part is those comes from those not rewiring with which is upper bounded by $k$. The other part comes form those rewiring from other vertexes which is a random variable with binomial distribution $\operatorname{Bin}((n-1) k p, 1 /(n-1))$. Then let $\mu=k p, \lambda=s-\mu$. By Chernoff's bounds, we have

$$
\operatorname{Pr}(d(v) \geq s) \leq \exp \left(-\frac{\lambda^{2}}{2(\mu+\lambda / 3)}\right) \text {. }
$$

Setting

$$
\lambda=\left(\frac{\alpha}{3}+\sqrt{\frac{\alpha^{2}}{9}+2 c \alpha}\right) p \log n .
$$

it is easy to see $\operatorname{Pr}(d(v) \geq s) \leq n^{-\alpha}$.

Hence

$$
\operatorname{Pr}\left(\Delta>(2+p) k+\left(\frac{\alpha}{3}+\sqrt{\frac{\alpha^{2}}{9}+2 c \alpha}\right) p \log n\right) \leq n^{-\alpha+1} \rightarrow 0 .
$$

Thus the maximum degree of $W S(n, k, p)$ has an upper bound

$$
\left.(2+p) k+\left(\frac{\alpha}{3}+\sqrt{\frac{\alpha^{2}}{9}+2 c \alpha}\right) p \log n\right)
$$

On the other hand, for any $\alpha \leq 1$ the assertion is obvious. Hence the maximum degree of $W S(n, k, p)$ has an upper bound

$$
(2+p) c \log n+\left(\frac{1}{3}+\sqrt{\frac{1}{9}+2 c}\right) p \log n .
$$

\section{Network Dynamics and Spectral Analysis of Watts-Strogats Network}

In a multi-agent system, the information exchange network between agents can be described by a graph $G=(V, E)$ with vertices $V$ and edges $E$. Each vertex is denoted by $i \in V=\{1,2, \ldots, n\}$, which stands for agents, and each edge is denoted by $e_{i j}=(i, j) \in E \subseteq V \times V$ for an information exchange link between agents. Let $A=\left(a_{i j}\right)$ be the adjacency matrix of the graph $G$. In this paper, we

assume that all graphs are undirected, which means that the adjacency matrices are symmetric.

Let $d_{i}=\sum_{j} a i_{j}$ be the degree of the vertex $i$, and $D=\operatorname{diag}\left(d_{1}, \ldots, d_{n}\right)$ is called the degree diagonal

matrix of the graph $G$. The Laplacian matrix of the graph $G$ is then defined by

$L(G)=D-A$.

Let $x_{i} \in \mathfrak{R}$ be the state of agent $i$, which is a real number assigned to the vertex $i$, then the network dynamics with the linear consensus protocol [8]

$\dot{x}(t)=-L x(t-\tau)=-(D-A) x(t-\tau)$.

solves the consensus problem, i.e,

$\lim _{t \rightarrow \infty} x_{1}(t)=\ldots=\lim _{t \rightarrow \infty} x_{n}(t)$.

The Laplacian matrix $L$ of a graph is a symmetric and diagonal dominant matrix, so \$L\$ is semi-positive definite, and again, by the definition of the Laplacian $L$, the row-sums are all zeros, so $L$ has the eigenvector $\mathbf{1}=(1,,,,, 1)^{\mathbf{T}}$ with zero eigenvalue. The eigenvalues of $L$ are denoted by

$0=\lambda_{1} \leq \lambda_{2} \leq \ldots \leq \lambda_{n}$.

In the consensus problem without time delay, the system reaches consensus if and only if $\lambda_{2}>0$ [7] and the second smallest eigenvalue $\lambda_{2}$ is the measure of speed of consensus [10].Furthermore the eigenvalue $\lambda_{n}$, which is also called the spectral radius of the graph, is served as a measure of robustness of the linear consensus protocol [8] which is given in the following 
lemma.

Lemma 3: Let $G$ be an arbitrary graph and $\Delta(G)$ be the maximum degree. Then the consensus problem with delay on network $G$ reach consensus if and only if

$$
\tau<\frac{\pi}{2 \lambda_{n}}
$$

where $\lambda_{n}$ is the spectral radius of the graph $G$ and $\tau$ is the time delay parameter.

Therefore, to analyze the consensus problems with the linear consensus protocol is the spectral analysis of Laplacian matrix of the network topology indeed. In the following, we denote $\lambda_{n}$ as $\lambda_{\max }$ to emphasize that it is the largest eigenvalue of the Laplacian matrix. In fact, $\lambda_{\max }$ is closely related to the maximum degree of the correspondent graph and we have the following lemma.

Lemma 4: Let $G$ be an arbitrary graph, $\lambda_{\max }$ be the maximum eigenvalue of the Laplacian matrix(i.e. spectral radius) and $\Delta(G)$ be the maximum degree. Then we have

$$
\lambda_{\max } \leq 2 \Delta(G) \text {. }
$$

Proof:

Since $\lambda_{\max }$ is an eigenvalue, then suppose $\mathbf{x}=\left(\mathrm{x}_{1}, \mathrm{x}_{2}, \ldots, \mathrm{x}_{\mathrm{n}}\right)$ be the corresponding eigenvector, and let $\left|\mathrm{x}_{\mathrm{i}}\right|=\max \left\{\left|\mathrm{x}_{-} \mathrm{j}\right| \mathrm{j} \mathrm{j}=1, \ldots, \mathrm{n} \backslash\right\}$, and $L=\left(l_{i j}\right) \quad$ be the Laplacian matrix. Then $\left|\lambda_{\max } x_{i}\right|=\sum_{j}\left|l_{i j} x_{j}\right| \leq l_{i i} x_{i}+\sum_{j \neq i}\left|l_{i j} x_{j}\right| \leq d_{i}\left|x_{i}\right|+\sum_{j \neq i}\left|x_{j}\right| \leq 2 d_{i}\left|x_{i}\right| \leq 2 \Delta\left|x_{i}\right|$.

Hence $\lambda_{\max } \leq 2 \Delta(G)$.

Then we are able to prove our main theorems as follows.

Theorem 1: Let $W S(n, c \log n, p)$ be the Watts-Stogatz small-world network with $n$ vertices, and parameters $c, p$. Then the maximum eigenvalue of the Laplacian matrix is almost surely upper bounded by

$$
(4+2 p) c \log n+\left(\frac{2}{3}+\sqrt{\frac{4}{9}+8 c}\right) p \log n
$$

Proof:

Remember that Lemma 2 gives the upper bound of maximum degree of small-world network while Lemma 4 gives the relationship between spectral radius and maximum degree. The theorem follows directly by combining Lemmas 2 and 4.

Theorem 2: Consider the network topology of Watt-Strogatz network consensus problem with parameters $n$,clogn,p, then the sufficient condition of reaching consensus with time delay $\tau$ is

$$
\tau<\frac{\pi}{4 p \log n}\left(c+\frac{2}{p}+\frac{1}{3}+\sqrt{\frac{1}{9}+2 c}\right)^{-1}
$$

Proof:

Lemma 3 shows that the sufficient and necessary condition for arbitrary graph is $\tau<\pi / 2 \lambda_{n}$, combining the result with Theorem 1 we derive our desired result.

Theorem 2 provides a general closed-form analytical result that builds the relationship between network topological characteristic parameters like $n$ and $p$ and the network dynamical characteristic parameter of time delay. In particular, applying theorem 2 we can control the time delay given the network while we can also design the required small-world topology given the target time delay. 


\section{Simulation Result and Conclusion}

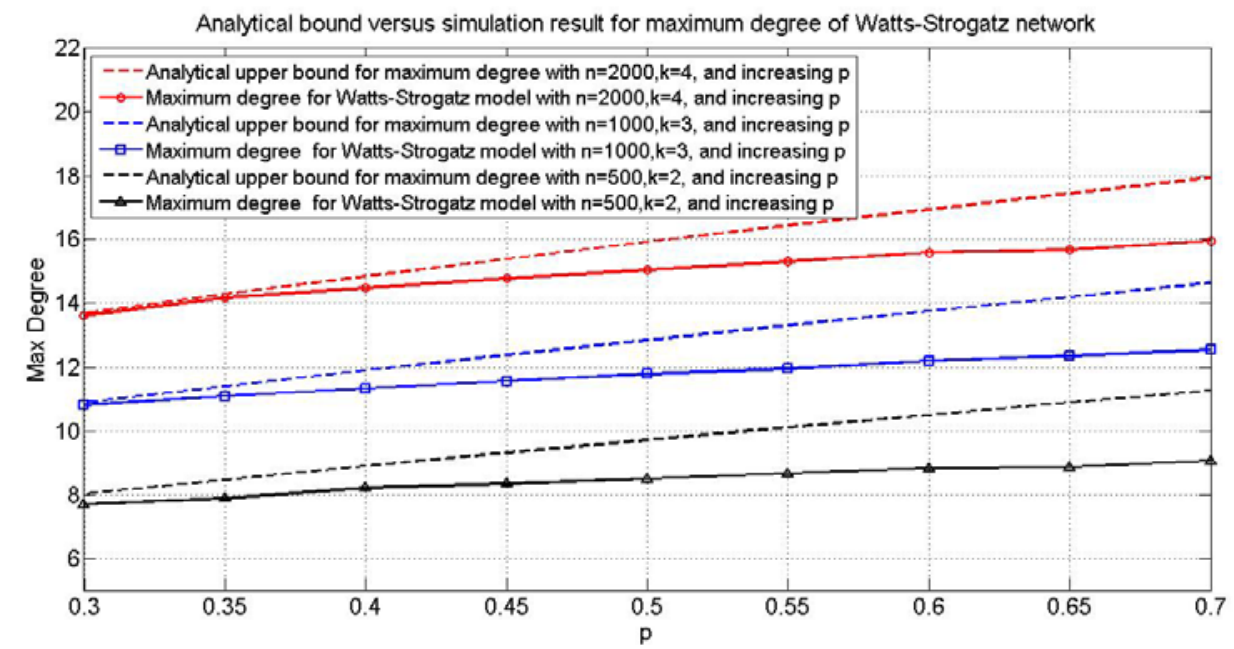

Fig. 2 Analytical results versus simulation results for maximum degree of Watts-Strogatz network with increasing $\mathrm{p}$, which shows close fitness of our analytical upper bound

In this part, experimental simulations will be presented to verify our theoretical analysis, and show the effectiveness of our result. We test the theoretical result in last section. In Figure 2 and Figure 3, we compare the spectral radius and the upper bound with maximum degree of Watts-Strogatz network. Here we compare the results with $n=500,1000,2000$ and $k=2,3,4$ respectively, and with $p$ increasing from 0.3 to 0.7 . To eliminate the influence of randomness, we take average from 2000 samples each. In Figure 2, analytical results in lemma 2 is applied to compare with the simulation results which shows very good fitness of our analytical upper bound.

In Figure 3, analytical results in theorem 1 is applied to compare with the simulation results and also verifies our results in the theorem. These results imply that our analysis and close-form formula behave effectively in the design of the efficient small-world network and time delay robust.

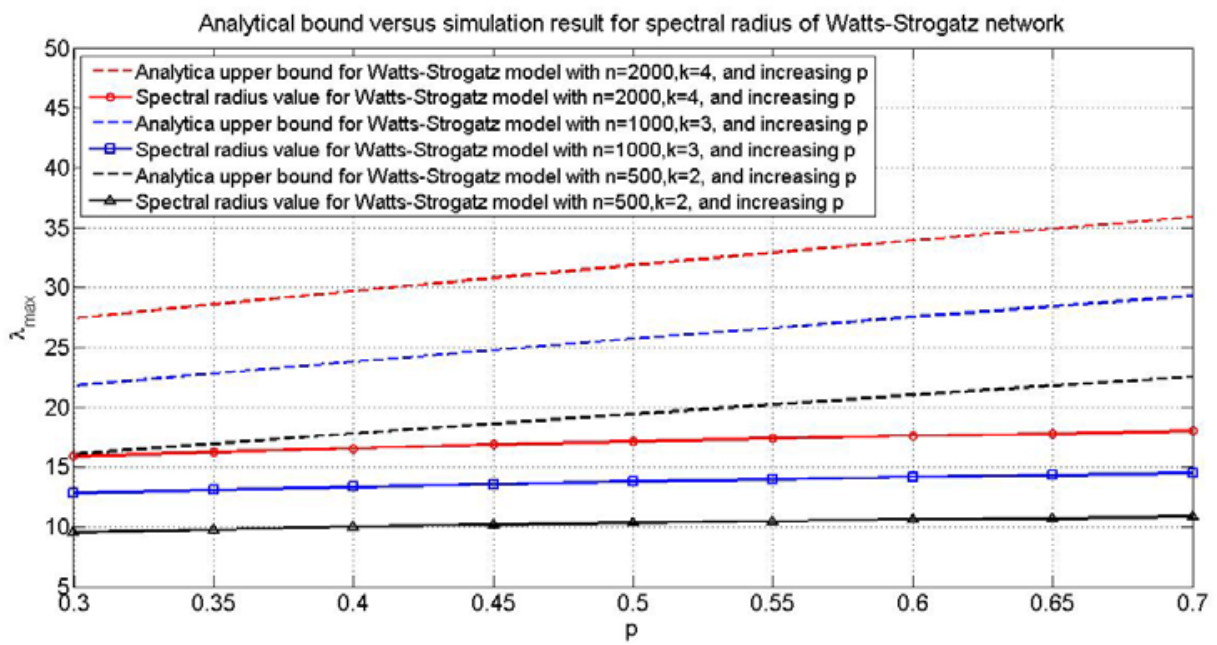

Fig. 3 Analytical results versus simulation results for spectral radius of Watts-Strogatz network with increasing $\mathrm{p}$, which verify our analytical upper bound

In this paper, we study the consensus problem on small-world network with time delay. In particular, we give the sufficient condition of the consensus problem reaching consensus base on a rigorous spectral analysis for the

Laplacian matrix of small-world networks. Closed-form upper bound for the spectral radius for Watts-Strogatz type small-world network is presented. These general closed-form analytical results build the relationship between network topological characteristic and the network dynamical 
characteristic of time delay. Applying these results we can control the time delay given the network while we can also design the required small-world topology given the target time delay. Simulation results verify our analysis and close-form formula behave effectively in the design of the efficient small-world network with time delay robustness.

\section{Acknowledgement}

In this paper, the research was supported by the Ministry of Science and Technology under the grant MOST 103-2221-E-002-022-MY3, the Major Research Plan of the National Natural Science Foundation of China under Grant 91118008, and by Key Projects in the National Science \& Technology Pillar Program during the Twelfth Five-year Plan Period under Grant 2014BAC16B01.

\section{References}

[1] S. Milgram, The small world problems, Psychology Today, 1967,1:62-67.

[2] D. J. Watts and S. H. Strogatz, Collective dynamics of "small world networks", Nature, 393,1998,440-442.

[3] M. E. J.Newman, The structure and function of complex networks, SIAM Review, 45,2003, 167-245.

[4] L.Gu, H.L.Huang. and X.D.Zhang, The Clustering Coefficient and the Diameter of Small-world Networks, Acta Mathematica Sinca, English Series,2013,29(1):199-208.

[5] A.Jadbabaie, J.Lin and A.D.Morse, Coordination of groups of mobile autonomous agents using nearest neighbor rules, IEEE Trans. On Automatic Control, 48,2003,988-1001.

[6] J.A. Fax and R. M. Murray, Information flow and cooperative control of vehicle formtations, IEEE Trans. On Automatic Control, 49,2004, 1465-1476.

[7] R. O. Saber and R.M. Murray, Consensus protocols for networks of dynamic agents, \{ $\backslash$ it Proceeeding of American Control Conference 2003, 2:951-956.

[8] R. Olfati and R. M. Murray, Consensus problems in networks of agents with swtiching topology and time-delays, IEEE Trans. on Automatic and Control, 49,2004, 1520-1533.

[9] W.Ren, R.W. Beard and E.M.Atkins, A survey of consensus problems in multi-agent coordination, Proceedings of American Control Conference 2005, 1859-1864.

[10] L. Gu, X. D. Zhang and Q. Zhou, Consensus and synchronization problems on small-world networks, J. Math. Phys., 51(8),2010, 082701.

[11] R.Olfati-Saber, Ultrafast consensus in the small-world networks, in \{lit Proceedings of Americn Control Conference 2005, 2371-2378.

[12] D. J. Watts, Small Worlds: The Dynamics of Networks between Order and Randomness, Princeton University Press,2003. 